\title{
Association of paraoxonase 1 gene polymorphisms with risk of Parkinson's disease: a meta-analysis
}

\author{
Received: 19 March 2004 / Accepted: 24 May 2004/Published online: 4 August 2004
}

(C) The Japan Society of Human Genetics and Springer-Verlag 2004

\begin{abstract}
Paraoxonase1 (PON1) gene polymorphisms were implicated as risk factors for Parkinson's disease (PD), but the results of case-control studies that investigated these associations were controversial. In order to provide an answer to these contradictory results, a metaanalysis of all available studies relating the PON1-55M/ $\mathrm{L}$ and PON1-192Q/R polymorphisms to the risk of developing PD was conducted. The racial descent of the populations in these studies was Caucasian and Asian. The meta-analysis revealed that there was an association of the PON1-55M allele and the risk of developing PD relative to the $\mathrm{L}$ allele: fixed effects pooled odds ratio $(\mathrm{OR})=1.32[95 \% \mathrm{CI}(1.10-1.59)]$. In addition, there was evidence of association for the genotypic contrast PON1-55MM + LM relative to PON1-55LL: fixed effects $\mathrm{OR}=1.50$ [95\%CI (1.16-1.95)]. There was no significant association between PON1-192Q/R alleles and risk of developing $\mathrm{PD}: \mathrm{OR}=1.09 \quad[95 \% \mathrm{CI}$ $(0.93-1.26)]$. There was no evidence for an association between the genotypic contrasts of PON1-192 and development of PD. The heterogeneity between studies and the publication bias were not significant $(P \geq 0.10)$ in either polymorphism. Therefore, the pooled results of the meta-analysis supported that there was an association between PON1-55M/L polymorphism and PD and that PON1-192Q/R polymorphism was unlikely to be a major risk factor for susceptibility to PD.
\end{abstract}

\section{E. Zintzaras}

Department of Biomathematics,

University of Thessaly School of Medicine,

Papakyriazi 22, Larissa 41222, Greece

G. M. Hadjigeorgiou $(\bowtie)$

Neurogenetics Unit, Department of Neurology,

University Hospital of Larissa,

University of Thessaly School of Medicine,

Papakyriazi 22, Larissa 41222, Greece

E-mail: gmhadji@med.uth.gr

Tel.: + 30-697-2862909

Fax: + 30-2410-611097
Keywords Paraoxonase PON1 Polymorphism ·

Parkinson's disease $\cdot$ Meta-analysis

\section{Introduction}

Parkinson's disease (PD) is one of the most common neurodegenerative disorders among the elderly and is characterized clinically by bradykinesia, rigidity, and resting tremor. Despite being the subject of intense study, the pathogenesis of PD remains unclear. In recent years, however, there has been increasing evidence to support a role for both genetic and environmental factors in its cause (Tan et al. 2000; Warner and Schapira 2003).

Epidemiological studies have found associations of PD with suspected pathways of pesticide exposure (Le Couteur et al. 1999). Paraoxonase is a detoxifying enzyme, or pesticide-metabolizing enzyme, which may predispose to $\mathrm{PD}$. In particular, the paraoxonase1 (PON1) gene product, serum paraoxonase, is an arylesterase synthesized mainly in the liver that hydrolyzes organophosphates such as pesticides, neurotoxins, and arylesters (Gowan et al. 2001). PON1 belongs to a gene cluster consisting of three highly homologous genes mapping to human chromosome 7q21.3 (Mochizuki et al. 1998). Expression of PON1 is controlled by both genetic and environmental factors (Carmine et al. 2002). Two frequent polymorphisms in the PON1 gene are the methionine ( $\mathrm{M}$ allele)-leucine ( $\mathrm{L}$ allele) interchange at position 55, and the glutamine (Q allele)-arginine ( $\mathrm{R}$ allele) interchange at position 192.

It was postulated that the PON1-55M/L and the PON1-192Q/R polymorphisms in the PON1 gene were associated with an increased risk for PD. The PON1$55 \mathrm{M}$ allele was correlated with decreased mRNA and protein levels and therefore: (a) carriers of the PON1$55 \mathrm{M}$ allele may have an inherited defect in detoxification of environmental toxins, and (b) this allele constitutes one possible candidate for PD susceptibility (Adkins et al. 1993; Humbert et al. 1993; Carmine et al. 2002; Kelada et al. 2003). Compared to PON1-192Q allele 
carries, PON1-192R allele carriers showed higher activity towards paraoxon and chlorpyrilos oxon but lower activity towards diazoxon, soman, and sarin that might contribute to development of PD (Mackness et al. 1997; Kondo and Yamamoto 1998; Kelada et al. 2003).

A number of case-control studies investigated the association between PON1 polymorphisms and the risk of $\mathrm{PD}$, but the results were inconclusive. Some studies urged that the $55 \mathrm{M}$ variant of PON1 was a risk factor for developing PD (Carmine et al. 2002; Akhmedova et al. 2001; Kelada et al. 2003), whilst other studies reported no genetic association between the PON1-55M/L polymorphism and PD (Wang and Liu 2000; Kelada et al. 2003). Concerning the PON1-192Q/R polymorphism, there were studies that did not establish association between this polymorphism and susceptibility to PD (Akhmedova et al. 1999; Taylor et al. 2000; Wang and Liu 2000; Kelada et al. 2003); however, one study urged that the 192R variant was a risk factor for developing PD (Kondo and Yamamoto 1998).

In our attempt to provide an answer to these controversial results, a meta-analysis of all available studies relating the PON1-55M/L and PON1-192Q/R polymorphisms to the risk of developing PD was conducted. In the meta-analysis, the estimates of the genetic association of each individual study and a pooled estimate of this association were obtained. In addition, the heterogeneity between studies and the publication bias were investigated. The PON1-55M/L and the PON1-192Q/R polymorphisms were analyzed separately.

\section{Material and methods}

\section{Selection of studies}

All studies that investigated the association of the PON1-55M/L and PON1-192Q/R polymorphisms with the development of PD and published before December 2003 were considered in the meta-analysis. The studies were identified by extended computer-based searches of the PubMed database. As a search criterion, we used various combinations of the following terms: paraoxonase, PON1, and Parkinson's disease.

Case-control studies that determined the distribution of PON1-55M/L and/or PON1-192Q/R genotypes in cases with PD and in a control group were eligible for inclusion in the meta-analysis. Cases were considered as PD after diagnosis using published criteria. The control group consisted of healthy subjects free of PD. Studies based on pedigree data were excluded, since they did not investigate association but linkage (Weir 1996; Ntais et al. 2003).

\section{Data extraction}

From each study, the following information was extracted: first author, journal, year of publication, racial descent of study population, demographics, matching, validity of genotyping method, and the number of cases and controls for each PON1-55M/L and PON1-192Q/R genotypes. Studies of different racial descent were considered separately. Allele frequencies were calculated, for cases and controls, from the corresponding genotype distributions.

\section{Meta-analysis}

Due to possible statistical inconsistencies and design differentiations between the studies, the significance of the association between the alleles of PON1-55 (M and $\mathrm{L}$ alleles), PON1-192 (Q and R alleles) and risk of having PD was tested for each study. Then the association of the PON1-55MM genotype with the risk of PD relative to the PON1-55LL genotype and the association of the PON1-192RR genotype with the risk of PD relative to the PON1-192QQ genotype were investigated. Contrasts of homozygotes versus the remaining genotypes were also assessed. All associations were indicated as odds ratios (OR) with the corresponding 95\% confidence interval (CI). Based on individual ORs, fixed effects pooled OR and random effects pooled OR were estimated.

Heterogeneity between studies in terms of degree of association was tested using the Q-statistic, which is a weighted sum of squares of the deviations of individual study OR estimates from the overall estimate (Cochran 1954). When ORs are homogeneous, Q follows a chisquared distribution with $r-1$ ( $r$ is the number of studies) degrees of freedom (d.f.). If $P<0.10$, then heterogeneity was considered significant. Heterogeneity was quantified with the $I^{2}$ metric $\left(I^{2}=(\mathrm{Q}-\right.$ d.f. $\left.) / \mathrm{Q}\right)$, which is independent of the number of studies in the meta-analysis (Higgins et al. 2003). $I^{2}$ takes values between $0 \%$ and $100 \%$ with higher values denoting greater degree of heterogeneity $\left(I^{2}=0-25 \%\right.$ : no heterogeneity; $I^{2}=25-50 \%$ : moderate heterogeneity; $I^{2}=50-75 \%$ : large heterogeneity; $I^{2}=75$ $100 \%$ : extreme heterogeneity). In addition, the null hypothesis of no allele or genotype effect for all studies was tested using the U-statistic: $U=\left(\sum \theta_{i} w_{i}\right)^{2} / \sum w_{i}$, where $\theta_{i}=\operatorname{lnOR}$ of the $i$ th study and $w_{i}=1 / \operatorname{var}\left(\theta_{i}\right)$. Under the null hypothesis, $U$ follows the chi-squared distribution with one d.f. (Lau et al. 1997; Whitehead 2002). The pooled ORs were estimated using fixed and random effects models. In the fixed effects model, the true OR of a contrast is considered to be the same for all studies, and the observed variability is due to chance. The random effects model allows the between-study variability to be accounted for in the pooled estimate and its standard error and tends to provide wider confidence intervals. When there was heterogeneity between studies, then the appropriated pooled OR was estimated using the random effects model (Lau et al. 1997; Whitehead 2002). Adjusted estimates of OR were considered whenever it was possible in a separate analysis. Separate pooled ORs for each race were also 
estimated. A cumulative meta-analysis and recursive meta-analysis were carried out in order to evaluate the trend of pooled OR for the allele contrasts $\mathrm{M}$ versus $\mathrm{L}$ and $\mathrm{R}$ versus $\mathrm{Q}$ in time (Lau et al. 1992, 1997; Ioannidis 1998; Whitehead 1997). Publication bias was tested for allele contrast using the $\alpha$-statistic (Ioannidis 1998; Whitehead 2002).

Analysis was carried out using SAS macros provided by Medical and Pharmaceutics Statistics Research Unit, University of Reading (Reading, UK) (Whitehead 2002).

\section{Results}

Eligible studies

Four studies investigating the association between PON1-55M/L polymorphism (Wang and Liu 2000; Akhmedova et al. 2001; Carmine et al. 2002; Kelada et al. 2003) and PD and five studies investigating the association between PON1-192Q/R polymorphism and PD were identified (Kondo and Yamamoto 1998; Akhmedova et al. 1999; Taylor et al.2000; Wang and Liu 2000; Kelada et al. 2003) (Table 1). Two studies (Wang and Liu 2000; Kelada et al. 2003) investigated both the PON1-55M/L and PON1-192Q/R polymorphisms. One study (Akhmedova et al. 2001) presented results for both $\mathrm{PON}-55 \mathrm{M} / \mathrm{L}$ and PON1-192Q/R; however, it was implied that the PON1-192 cases and controls were a subgroup of another eligible study (Akhmedova et al. 1999) and therefore PON1-192 data of that study were not considered in the subsequent analysis.

Concerning the $\mathrm{PON} 1-55 \mathrm{M} / \mathrm{L}$ polymorphism, in three studies (Akhmedova et al. 2001; Carmine et al. 2002; Kelada et al. 2003), the subjects were Caucasians, whilst in one study, the subjects were Asians (Wang and Liu 2000) (Table 1). In all four studies (Wang and Liu 2000; Akhmedova et al. 2001; Carmine et al. 2002; Kelada et al. 2003), unrelated idiopathic PD patients were included; in one study (Carmine et al. 2002) $28.9 \%$ of cases had affected relatives. PD patients were diagnosed according to valid diagnostic criteria. The average age at onset was estimated to vary from 51 to 61 years across studies, and the average age varied from 51 to 67 years. Controls were healthy individuals; in two studies (Wang and Liu 2000; Akhmedova et al. 2001), it was reported that they were unrelated individuals, whilst in one study (Kelada et al. 2003), it was reported that they had no past history of PD or other neurodegenerative disorder. In two studies (Wang and Liu 2000; Kelada et al. 2003), it was denoted that there was matching for gender and age. Generally, gender was not evenly distributed. Mean age of controls varied from 38 to 77 years.

Concerning the PON1-192Q/R polymorphism, in three studies (Taylor et al. 2000; Akhmedova et al. 2001; Kelada et al. 2003), the subjects were Caucasians, whilst in two studies, the subjects were Asians (Kondo and Yamamoto 1998; Wang and Lin 2000) (Table 1). In three studies, it was stated that the cases were unrelated idiopathic PD patients (Akhmedova et al. 1999; Wang and Liu 2000; Kelada et al. 2003). One study did not report diagnostic criteria (Taylor et al. 2000). Three studies (Kondo and Yamamoto 1998; Akhmedova et al. 1999; Taylor et al. 2000) did not report age at onset, one study did not report gender (Akhmedova et al. 1999), and one study (Taylor et al. 2000) did not report demographic data. Controls were healthy individuals; in two studies (Kondo and Yamamoto 1998; Wang and Liu 2000), it was reported that they were unrelated individuals. In three studies (Taylor et al. 2000; Wang and Liu 2000; Kelada et al. 2003), it was denoted that there was matching for gender and age, and in one study (Akhmedova et al. 1999), there was matching only for age (Table 1). One study investigated the association between pesticide exposure, PD, and polymorphism (Taylor et al. 2000). Data of all subjects from that study, exposed and nonexposed, were considered in the meta-analysis, since pesticide exposure had no significant effect (Taylor et al. 2000).

All studies used the validated genotyping method: PCR analysis followed by endonuclease digestion (Kondo and Yamamoto 1998; Akhmedova et al. 1999; Wang and Liu 2000; Taylor et al.2000; Akhmedova et al. 2001; Carmine et al. 2002; Kelada et al. 2003).

\section{Summary statistics}

In total, the PON1-55 studies included 561 cases and 757 controls. In both PD and control groups, allele L was the most frequent, and the prevalence of the LL genotype was the highest, whilst the prevalence of the MM genotype was the lowest (Tables 2, 3 and Fig. 1).

The PON1-192 studies included 706 cases and 913 controls. In both PD and control groups, allele $\mathrm{Q}$ was the most frequent, the prevalence of $\mathrm{QR}$ was the highest, whereas the RR genotype was the lowest. (Tables 2, 3 and Fig. 1). In all studies, distribution of genotypes in the control group was in Hardy-Weinberg (H-W) equilibrium $(P \geq 0.05)$ (Weir 1996; SAGE 2003).

\section{Contrast of alleles}

In investigating the association of allele PON1-55M and the risk of developing PD relative to allele PON1-55L, the meta-analysis showed that there was no heterogeneity $\left(P=0.20, I^{2}=35 \%\right)$ between the four studies (Kelada et al. 2003; Akhmedova et al. 2001; Wang and Liu 2000; Kondo and Yamamoto 1998) and that the ORs of the studies were significantly different from zero. Therefore, the pooled OR for fixed effects was considered, which was OR $=1.32$ [95\% CI $(1.10-1.59)]$, indicating that the PON1-55M allele might be responsible for developing PD. The meta-analysis on the Caucasian population (Kelada et al. 2003; Carmine et al. 2002; Akhmedova et al. 2001) produced similar results: $\mathrm{OR}=1.34 \quad[95 \% \mathrm{CI}(1.11-1.62)]$, whereas, the Asian 


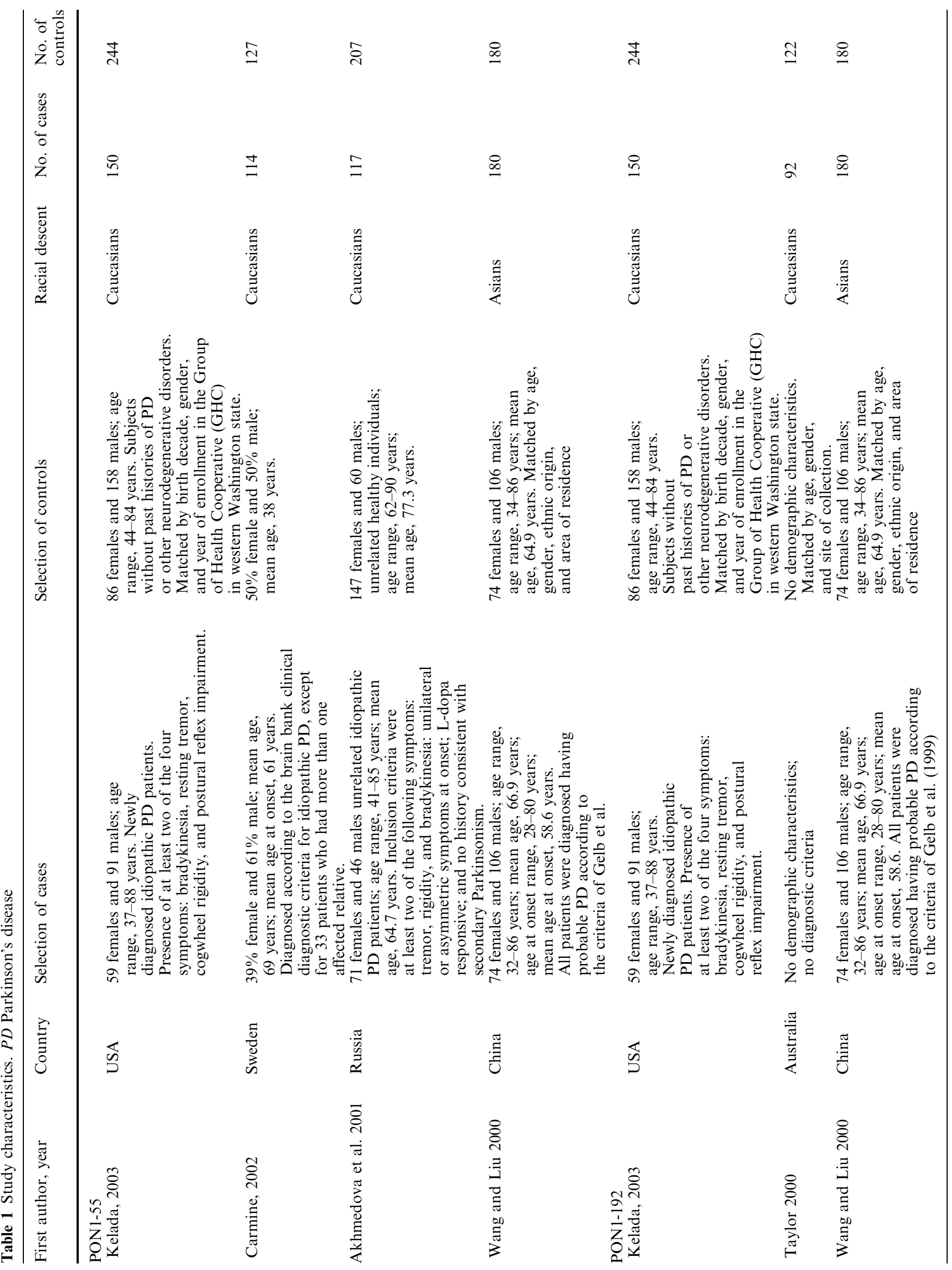


study (Wang and Liu 2000) revealed no association: $\mathrm{OR}=1.07[95 \% \mathrm{CI}(0.52-2.20)]$ (Tables 4, 5).

Overall, there was no significant association between PON1-192Q/R polymorphism and risk of developing PD: $\quad \mathrm{OR}=1.09 \quad[95 \% \mathrm{CI} \quad(0.93-1.26)]$. Heterogeneity between studies was not significant $\left(P=0.18, I^{2}=37 \%\right)$. In the Asian population (Kondo and Yamamoto 1998; Wang and Liu 2000), heterogeneity was not significant $\left(P=0.13, I^{2}=56 \%\right)$, and the fixed effects pooled OR was 1.25 [95\% CI $(1.01,1.54)]$, which could be considered rather marginal. Therefore, there was no indication that the PON1-192Q/R polymorphism plays a role in the development of PD (Tables 4, 5).

\section{Contrast of genotypes}

There was evidence of association of the PON1-55MM genotype with the risk of PD relative to the PON1-55LL genotype. Since there was no heterogeneity $(P=0.29$, $I^{2}=0 \%$ ) between the studies, the fixed effects pooled OR was considered: $\mathrm{OR}=1.64$ [95\%CI (1.10-2.46)]. There was significant association of the PON1-55MM + LM genotype with the risk of PD relative to the LL genotype; there was no heterogeneity $\left(P=0.20, I^{2}=35 \%\right)$ between the studies (Kelada et al. 2003; Akhmedova et al. 2001; Wang and Liu 2000; Kondo and Yamamoto $1998)$ and the fixed effects OR was 1.45 [95\%CI (1.13, 1.86)]. Meta-analysis on the Caucasian data (Kelada et al. 2003; Carmine et al. 2002; Akhmedova et al. 2001) showed the significance of PON1-55MM and MM + LM genotypes in developing PD: $\mathrm{OR}=1.66[95 \% \mathrm{CI}(1.10$, $2.49)]$ and $\mathrm{OR}=1.50$ [95\% CI $(1.16,1.95)]$, respectively. Meta-analysis on the Asian study (Wang and Liu 2000) showed no significant association between any genotypes and the risk of PD (Tables 4, 5).

Overall, there was no evidence for an association between the genotypic contrasts of PON1-192 and PD. There was no heterogeneity $\left(P \geq 0.10, I^{2}=0-56 \%\right)$ between studies for all genotypic contrasts. The same pattern applied also to the Caucasian population. In the Asian data (Wang and Liu 2000; Kondo and Yamamoto 1998), there was marginal evidence of association of the PON1192RR genotype with the risk of PD relative to the $\mathrm{QR}+\mathrm{QQ}$ genotype: $\mathrm{OR}=1.35 \quad$ [95\% CI $\quad(1.01-1.81)]$ (Tables 4,5$)$. Since all studies provided unadjusted and unstratified estimates, adjusted pooled OR were not calculated.

Bias

For the PON1-55M/L polymorphism, in cumulative and recursive meta-analysis, the magnitude of fixed effects pooled $\mathrm{OR}$ for alleles $\mathrm{M}$ versus $\mathrm{L}$ was relative stable after the first publication: $\mathrm{OR}=1.07$ in $2000, \mathrm{OR}=1.54$ in $2001, \mathrm{OR}=1.51$ in 2002 , and $\mathrm{OR}=1.32$ in 2003 . For the PON1-192Q/R polymorphism in cumulative and recursive meta-analysis, fixed effects pooled OR for alleles $\mathrm{R}$ 
Table 2 Distribution of PON1-55 genotypes and frequency of alleles for patients with Parkinson's disease (PD) and patients without PD (controls). The odds ratios (OR) of PD with the corresponding 95\% confidence intervals (CI) are demonstrated

\begin{tabular}{|c|c|c|c|c|c|c|c|c|c|c|c|c|}
\hline \multirow[t]{3}{*}{ Study } & \multirow[t]{3}{*}{ Race } & \multicolumn{6}{|c|}{ Distribution of PON1-55 genotypes } & \multicolumn{4}{|c|}{ Frequency of PON1-55 alleles } & \multirow[t]{3}{*}{$\mathrm{OR}_{\mathrm{M} \text { versus } \mathrm{L}}(95 \% \mathrm{CI})$} \\
\hline & & \multicolumn{2}{|l|}{ LL } & \multicolumn{2}{|l|}{ LM } & \multicolumn{2}{|c|}{ MM } & \multicolumn{2}{|l|}{$\mathrm{L}$} & \multicolumn{2}{|l|}{ M } & \\
\hline & & PD & Control & PD & Control & PD & Control & PD & Control & PD & Control & \\
\hline Kelada & Caucasian & 60 & 105 & 70 & 104 & 20 & 34 & 190 & 314 & 110 & 172 & $1.06(0.79-1.43)$ \\
\hline Carmine & Caucasian & 41 & 58 & 52 & 56 & 21 & 13 & 134 & 172 & 94 & 82 & $1.47(1.01-2.13)$ \\
\hline Akhmedova & Caucasian & 36 & 102 & 61 & 80 & 20 & 25 & 133 & 284 & 101 & 130 & $1.67(1.20-2.33)$ \\
\hline Wang & Asian & 165 & 166 & 14 & 13 & 1 & 1 & 344 & 345 & 16 & 15 & $1.07(0.52-2.20)$ \\
\hline
\end{tabular}

Table 3 Distribution of PON1-192 genotypes and frequency of alleles for patients with Parkinson's disease (PD) and patients without PD (controls). The odds ratios (OR) of PD with the corresponding $95 \%$ confidence intervals (CI) are demonstrated

\begin{tabular}{|c|c|c|c|c|c|c|c|c|c|c|c|c|}
\hline Study & Race & \multicolumn{6}{|c|}{ Distribution of PON1-192 genotypes } & \multicolumn{4}{|c|}{ Frequency of PON1-192 alleles } & $\mathrm{OR}_{\mathrm{R} \text { versus } \mathrm{Q}}(95 \% \mathrm{CI})$ \\
\hline Kelada & Caucasian & 81 & 121 & 57 & 97 & 12 & 24 & 219 & 339 & 81 & 145 & $0.87(0.63-1.20)$ \\
\hline Taylor & Caucasian & 42 & 63 & 45 & 46 & 5 & 13 & 129 & 172 & 55 & 72 & $1.02(0.67-1.55)$ \\
\hline Wang & Asinan & 25 & 26 & 85 & 88 & 70 & 66 & 135 & 140 & 225 & 220 & $1.06(0.78-1.43)$ \\
\hline
\end{tabular}

versus Q had a downward trend indicating that the lack of association is strengthen as the amount of data gets larger: $\mathrm{OR}=1.46$ in $1998, \mathrm{OR}=1.28$ in $1999, \mathrm{OR}=1.16$ in 2000 , and $\mathrm{OR}=1.09$ in 2003 . In both $\mathrm{PON} 1-55 \mathrm{M} / \mathrm{L}$ and $\mathrm{PON} 1-192 \mathrm{Q} / \mathrm{R}$ polymorphisms, the a-statistic for the allele contrasts was not significant: $\alpha=-0.28[95 \% \mathrm{CI}$ $(-3.88,3.33)]$ and $\alpha=-1.47$ [95\% CI $(-7.35,4.37)$, respectively, indicating the lack of publication bias.

\section{Discussion}

In recent years, interest in interactions between genetic and environmental factors that were implicated in the pathogenesis of PD has spurred a great number of association studies on polymorphisms of different genes (Tan et al. 2000). To date, no specific gene polymorphism has been unequivocally associated with PD (Warner and Schapira 2003). This was also true for PON1-55M/L and PON1-192Q/R polymorphisms. The present meta-analysis included four studies for the PON1-55M/L polymorphism with 1318 genotyped subjects, and five studies for the PON1-192Q/R polymorphism with 1512 genotyped subjects. The overall data demonstrated that the PON1-55M/L polymorphism could be a risk factor for PD. This was indicating by the significant association of allele $\mathrm{M}$, or $\mathrm{MM}+\mathrm{ML}$ genotype (dominant model), with an increased risk for
Fig. 1 Frequency of alleles and genotypic prevalence for both PON1 polymorphisms for the Parkinson's disease group (PD) and the control group [bars indicate $95 \%$ confidence intervals $(\mathrm{CI})]$

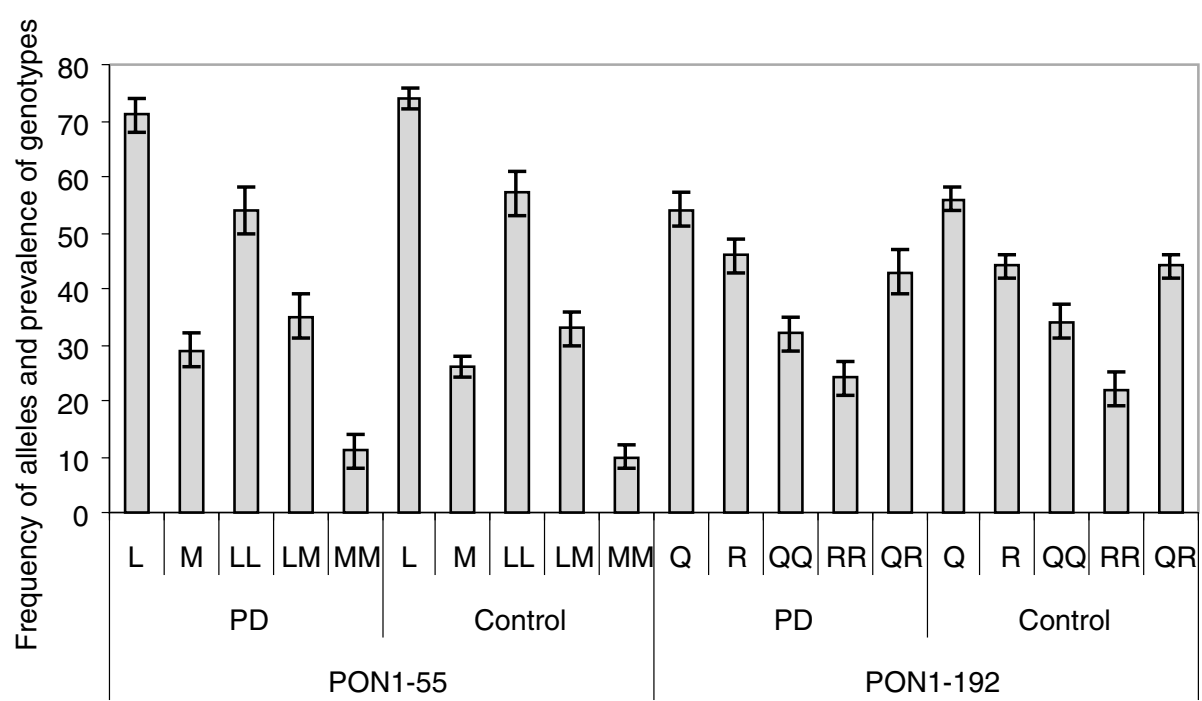


Table 4 Fixed and random pooled odds ratios (ORs) for allelic and genotypic contrasts for investigating the association between PON1-55 polymorphisms and the risk of Parkinson's disease (PD).
The significance ( $P$ value) of heterogeneity ( $Q$ test), the $I^{2}$ metric (when negative is set to zero), and of uniformity of estimates ( $U$ test) are also shown. $C I$ confidence intervals, n.a. not applicable

\begin{tabular}{|c|c|c|c|c|c|c|}
\hline Race & Contrast & $\begin{array}{l}\text { Fixed effects, } \\
\text { OR }(95 \% \mathrm{CI})\end{array}$ & $\begin{array}{l}P \text { value, } \\
Q \text { test }\end{array}$ & $I^{2}(\%)$ & $\begin{array}{l}P \text { value, } \\
U \text { test }\end{array}$ & $\begin{array}{l}\text { Random effects, } \\
\text { OR }(95 \% \mathrm{CI})\end{array}$ \\
\hline \multirow[t]{4}{*}{ All } & $M$ versus $L$ & $1.32(1.10-1.59)$ & 0.20 & 35 & $<0.01$ & n.a \\
\hline & $\mathrm{MM}$ versus $\mathrm{LM}+\mathrm{LL}$ & $1.34(0.92-1.94)$ & 0.46 & 0 & 0.13 & n.a \\
\hline & MM versus LL & $1.64(1.10-2.46)$ & 0.29 & 0 & 0.02 & n.a \\
\hline & $\mathrm{MM}+\mathrm{LM}$ versus $\mathrm{LL}$ & $1.45(1.13-1.86)$ & 0.20 & 35 & $<0.01$ & n.a. \\
\hline \multirow{4}{*}{ Caucasian } & $M$ versus $L$ & $1.34(1.11-1.62)$ & 0.12 & 53 & $<0.01$ & $1.36(1.03-1.79)$ \\
\hline & $M M$ versus $L M+L L$ & $1.34(0.92-1.95)$ & 0.28 & 0 & 0.12 & n.a. \\
\hline & MM versus $L L$ & $1.66(1.10-2.49)$ & 0.16 & 45 & 0.02 & $1.70(0.99-2.94)$ \\
\hline & $\mathrm{MM}+\mathrm{LM}$ versus $\mathrm{LL}$ & $1.50(1.16-1.95)$ & 0.14 & 49 & $<0.01$ & n.a. \\
\hline \multirow[t]{4}{*}{ Asian } & M versus L & $1.07(0.52-2.20)$ & n.a. & n.a. & n.a. & n.a. \\
\hline & $\mathrm{MM}$ versus $\mathrm{LM}+\mathrm{LL}$ & $1.00(0.06-15.99)$ & n.a. & n.a. & n.a. & n.a. \\
\hline & MM versus LL & $1.01(0.06-16.15)$ & n.a. & n.a. & n.a. & n.a. \\
\hline & $\mathrm{MM}+\mathrm{LM}$ versus $\mathrm{LL}$ & $1.08(0.51-2.30)$ & n.a. & n.a. & n.a. & n.a. \\
\hline
\end{tabular}

development of PD relative to the allele $\mathrm{L}$ or to the $\mathrm{LL}$ genotype. This association holds true especially in subjects of Caucasian descent (Akhmedova et al. 2001; Carmine et al. 2002; Kelada et al. 2003). The study on the Asian population has shown no significant association between the PON1-55M/L polymorphism and PD (Wang and Liu 2000).

In contrast, the PON1-192Q/R polymorphism could not be considered a major risk factor for susceptibility to PD. In investigating the allelic or genotypic contrasts, we found no statistically significant heterogeneity between the studies and lack of publication bias in both PON1-55M/L and PON1-192Q/R polymorphisms. However, quantitatively heterogeneity varied from no to moderate heterogeneity. The nonsignificant heterogeneity or publication bias found in present meta-analyses might be due to a lack of power to detect it because of the limited number of studies.

In a recent meta-analysis of 43 studies investigating the association of PON1 and PON2 with coronary heart disease, moderate linkage disequilibrium between PON1-55M/L and PON1-192Q/R was reported only in one study (Wheeler et al. 2004). In present meta-analysis, linkage disequilibrium has been reported only in one study (Akhmedova et al. 2001) but without providing any data or the $D$ ' value, therefore this result might be dubious. Since the majority of studies in the present meta-analysis reported on only one polymorphism, analyses of haplotypes could not be provided. Although different genotypic contrasts were addressed, adjustments for multiple comparisons were not performed, as generally such allowances have not been applicable in meta-analysis. Moreover, the examined contrasts are highly correlated, and $P$-value adjustments (e.g., Bonferonni's) would be too stringent. In PON1-55M/L, there was no time trend in the results with association. In PON1-192Q/R, only the results of the earliest published study were significant.

The meta-analysis was based on unadjusted estimates. However, a more precise analysis could be performed if adjusted (by gender, age, age at onset) estimates were provided in the studies. The results of the meta-analysis depended on study design and inclusion criteria of cases and controls in each study (Lau et al.1997). In this meta-analysis, cases were well defined with similar criteria and controls had a relatively low diversity. Meta-analysis summary results are based on the synthesis of published case-control studies, thus

Table 5 Fixed and random pooled odds ratios (ORs) for allelic and genotypic contrasts for investigating the association between PON1-192 polymorphisms and the risk of Parkinson's disease

(PD). The significance ( $P$ value) of heterogeneity ( $Q$ test), the $I^{2}$ metric (when negative is set to zero), and of uniformity of estimates ( $U$ test), are also shown. $C I$ confidence interval, n.a. not applicable

\begin{tabular}{|c|c|c|c|c|c|c|}
\hline Race & Contrast & $\begin{array}{l}\text { Fixed effects, } \\
\text { OR }(95 \% \mathrm{CI})\end{array}$ & $\begin{array}{l}P \text { value, } \\
Q \text { test }\end{array}$ & $I^{2}(\%)$ & $\begin{array}{l}P \text { value, } \\
U \text { test }\end{array}$ & $\begin{array}{l}\text { Random effects, } \\
\text { OR }(95 \% \mathrm{CI})\end{array}$ \\
\hline \multirow[t]{4}{*}{ All } & $R$ versus $Q$ & $1.09(0.93-1.26)$ & 0.18 & 37 & 0.28 & $1.08(0.90-1.30)$ \\
\hline & $\mathrm{RR}$ versus $\mathrm{QR}+\mathrm{QQ}$ & $1.15(0.90-1.49)$ & 0.14 & 43 & 0.27 & $1.05(0.73-1.51)$ \\
\hline & RR versus QQ & $1.11(0.78-1.56)$ & 0.17 & 38 & 0.57 & $1.06(0.68-1.66)$ \\
\hline & $\mathrm{RR}+\mathrm{QR}$ versus $\mathrm{QQ}$ & $1.08(0.86-1.36)$ & 0.41 & 0 & 0.52 & n.a. \\
\hline \multirow[t]{4}{*}{ Caucasian } & $R$ versus $Q$ & $0.93(0.75-1.16)$ & 0.81 & 0 & 0.54 & n.a. \\
\hline & $\mathrm{RR}$ versus $\mathrm{QR}+\mathrm{QQ}$ & $0.70(0.42-1.18)$ & 0.75 & 0 & 0.18 & n.a. \\
\hline & RR versus QQ & $0.72(0.42-1.22)$ & 0.92 & 0 & 0.22 & n.a. \\
\hline & $\mathrm{RR}+\mathrm{QR}$ versus QQ & $0.99(0.75-1.30)$ & 0.51 & 0 & 0.95 & n.a. \\
\hline \multirow[t]{4}{*}{ Asian } & $R$ versus $Q$ & $1.25(1.01-1.54)$ & 0.13 & 56 & 0.04 & $1.25(0.91-1.71)$ \\
\hline & $\mathrm{RR}$ versus $\mathrm{QR}+\mathrm{QQ}$ & $1.35(1.01-1.81)$ & 0.19 & 0 & 0.04 & $1.34(0.92-1.97)$ \\
\hline & RR versus QQ & $1.52(0.96-2.40)$ & 0.16 & 0 & 0.07 & $1.52(0.80-2.88)$ \\
\hline & $\mathrm{RR}+\mathrm{QR}$ versus $\mathrm{QQ}$ & $1.33(0.87-2.04)$ & 0.25 & 0 & 0.19 & n.a. \\
\hline
\end{tabular}


better power is achieved to identify an effect of the polymorphisms under investigation. Failure to obtain statistical significance at the 0.05 level in the PON1-192 allele contrast may be due to a type II error, that is, the accumulated evidence was still underpowered to detect a small difference in the effect size. However, the pooled OR of the meta-analysis excluded with $95 \%$ certainty that carriers of the PON1-192R allele would have more than 1.25-fold increased odds for developing the disease.

In conclusion, the pooled results supported an association between the PON1-55M/L polymorphism and $\mathrm{PD}$ and the lack of association between the PON1192Q/R polymorphism and PD. However, in order to establish a strong association between PON1-55M/L and PD, a large number of studies with similar study designs providing subgroup data such as age at onset, duration of disease, and gender, are required. In addition, other probable genetic risk factors interacting with the PON1 polymorphisms should be investigated.

\section{References}

Adkins S, Gan KN, Mody M, La Du BN (1993) Molecular basis for the polymorphic forms of human serum paraoxonase/arylesterase: glutamine or arginine at position 191, for the respective A or B allozymes. Am J Hum Genet 52:598-608

Akhmedova S, Anisimov S, Yakimovsky A, Schwartz E (1999) Gln $\rightarrow$ Arg 191 polymorphism of paraoxonase and Parkinson's disease. Hum Hered 49:178-180

Akhmedova S, Yakimovsky A, Schwartz EI (2001) Paraoxonase 1 Met-Leu 54 polymorphism is associated with Parkinson's disease. J Neurol Sci 184:179-182

Carmine A, Buervenich S, Sydow O, Anvret M, Olson L (2002) Further evidence for association of the paraoxonase 1 (PON1) Met-54 allele with Parkinson's disease. Mov Disord 17:764-766

Cochran WG (1954) The combination of estimates from different experiments. Biometrics 10:101-129

Gowan J, Sinton CM, Varley AW, Wians FH, Haley RW, Munford RS (2001) Gene therapy to prevent organophosphate intoxication. Toxicol Appl Pharmacol 173:1-6

Higgins JP, Thompson SG, Deeks JJ, Altman DG (2003) Measuring inconsistency in meta-analyses. Br Med J 327:557-560

Humbert R, Adler DA, Disteche CM, Hassett C, Omiecinski C, Furlog CE (1993) The molecular basis of human serum paraoxonase activity polymorphism. Nat Genet 3:73-76

Ioannidis JP (1998) Effect of the statistical significance of results on the time to completion and publication of randomized efficacy trials. J Am Med Assoc 279:281-286
Kelada SN, Costa-Mallen P, Checkoway H, Viernes HA, Farin FM, Smith-Weller T, Franklin GM, Costa LG (2003) Paraoxonase 1 promoter and coding region polymorphisms in Parkinson's disease. J Neurol Neurosurg Psychiatry 74:545-548

Kondo I, Yamamoto M (1998) Genetic polymorphism of paraoxonase 1 (PON1) and susceptibility to Parkinson's disease. Brain Res 806:271-273

Lau J, Antman EM, Jimenez-Silva J, Kupelnick B, Mosteller F, Chalmers TC (1992) Cumulative meta-analysis of therapeutic trials for myocardial infarction. N Engl J Med 327:248-254

Lau J, Ioannidis JP, Schmid CH (1997) Quantitative synthesis in systematic reviews. Ann Intern Med 127:820-826

Le Couteur DG, McLean AJ, Taylor MC, Woodham BL, Board PG (1999) Pesticides and Parkinson's disease. Biomed Pharmacother 53:122-130

Mackness B, Mackness MI, Arrol S, Turkie W, Durrington PN (1997) Effect of the molecular polymorphisms of human paraoxonase (PON1) on the rate of hydrolysis of paraoxon. $\mathrm{Br}$ J Pharmacol 122:265-268

Mochizuki H, Scherer SW, Xi T, Nickle DC, Majer M, Huizenga JJ, Tsui LC, Prochazka M (1998) Human PON2 gene at 7q21.3: cloning, multiple mRNA forms, and missense polymorphisms in the coding sequence. Gene 213:149-157

Ntais C, Polycarpou A, Ioannidis JP (2003) Association of the CYP17 gene polymorphism with the risk of prostate cancer: a meta-analysis. Cancer Epidemiol Biomarkers Prev 12:120-126

SAGE (2003) Statistical Analysis for Genetic Epidemiology. Computer program package available from Statistical Solutions Ltd, Cork

Tan EK, Khajavi M, Thornby JI, Nagamitsu S, Jankovic J, Ashizawa T (2000) Variability and validity of polymorphism association studies in Parkinson's disease. Neurology 55:533-538

Taylor MC, Le Couteur DG, Mellick GD, Board PG (2000) Paraoxonase polymorphisms, pesticide exposure and Parkinson's disease in a Caucasian population. J Neural Transm 107:979-983

Wang J, Liu Z (2000) No association between paraoxonase 1 (PON1) gene polymorphism and susceptibility to Parkinson's disease in a Chinese population. Mov Disord 15:1265-1267

Warner TT, Schapira AHV (2003) Genetic and environmental factors in the cause of Parkinson's disease. Ann Neurol 53(Suppl 3):S16-S25

Weir BS (1996) Genetic data analysis II: methods for discrete population genetic data. Sinauer Associates, Sunderland

Wheeler JG, Keavney BD, Watkins H, Collins R, Danesh J (2004) Four paraoxonase gene polymorphisms in 11,212 cases of coronary heart disease and 12,786 controls: meta-analysis of 43 studies. Lancet 363:689-695

Whitehead A (1997) A prospectively planned cumulative metaanalysis applied to a series of concurrent clinical trials. Stat Med 16:2901-2913

Whitehead A (2002) Meta-analysis of controlled clinical trials. Wiley, Chichester 